\title{
An Approach to Physiotherapy for the Patient with Parkinson's Disease.
}

S. H. IRWIN-CARRUTHERS, M.S.A.S.P., Dip. Onderwys (Pretoria)

Lecturer in Physiotherapy, University of Stellenbosch.

The problem of how to approach treatment of the patient with Parkinson's disease has been one which has taxed physiotherapists for many years. If anything, we are faced by even larger numbers of these patients now than we were in the past. It may be that the cohort group theory ${ }^{1}$ accounts for this, and that a few generations hence Parkinson's disease will be to future physiotherapists what tabes dorsalis is to present-day workers - interesting academically but seldom encountered in clinical practice. At present, however, the patient with Parkinson's disease is a constantly present challenge to our resources as physiotherapists, and although few physicians would fail to refer these patients for physiotherapy, relatively few articles on the physiotherapy treatment of this disease have appeared in the literature. Those which have been published have, for the main part, been based upon modifications of proprioceptive neuromuscular facilitation techniques ${ }^{2,3,4}$ or on more traditional exercise regimes ${ }^{5,6,7}$ and are worth studying. The treatment of Parkinson's disease would, however, appear to go further than this, and many therapists are increasingly incorporating other neurophysiologically based approaches, as well as selected aspects of more traditional exercises in their treatment programmes. With the advent of $\mathrm{L}$-dopa, results have been accelerated and it is tempting to postulate that the drug alone may be responsible for the patient's improvement. Physicians at this hospital have, however, been adamant on the importance of physiotherapy and have not been in favour of clinical trials which, whilst proving the issue one way or another, might deprive certain patients of essential therapy. It does seem that physiotherapy has an important part to play in the rehabilitation of patients on L-dopa. L-dopa has greatly increased the functional potential of the Parkinson's patient, but is not the total answer to the problem. In many cases, dosage is limited by the development of side effects and physiotherapy has to assume a greater load in the further functional improvement of the patient. It has also been noted in the present trials that one or two patients whose general condition did not allow full active participation in the physical therapy programme showed correspondingly less response to L-dopa therapy. This is merely an observation, and many factors may have been involved, but it is suggestive.

\section{THE PROBLEMS}

It is not proposed to go into all the finer aspects of the clinical picture of the patient with Parkinson's disease. The basic problems are threefold - rigidity, akinesia and tremor. Rigidity

The rigidity appears related to a hyperactivity of the system. It has been suggested ${ }^{8}$ that damage (but not total destruction) of cells in the substantia nigra leads to release of its inhibitory control of the pallidum, so that the later structure remits facilitatory impulses to the reticular formation. The rigidity may be either plastic or cog-wheel in nature, and affects all muscle groups (as opposed to the selective hypertonus of spasticity). The patient does, however, tend to develop a generally flexed attitude in the later stages of the disease, flexion contractures sometimes even extending to the hands and feet. This could possibly be due to autofacilitation of the flexors as a result of secondary stretch caused by the sustained hypertonus. The extensors, on the other hand, would tend to be inhibited.

Functionally, the rigidity augments the akinesia in preventing movement. The patient cannot initiate movement, and movement through range is slowed and limited by hypertonus in the antagonists. The effort of movement may increase the hypertonus, so that reversal of the movement and reciprocal motion becomes almost impossible. Rigidity of tongue and jaw muscles retards speech chewing and swallowing - causing difficulties with feeding and sometimes being severe enough to cause drooling. Rigidity is hard to demonstrate in the facial muscles - and the mask-like appearance of the face may be more a result of akinesia.

Akinesia

The akinesia (or bradykinesia) is thought not to be due to the rigidity, but to be a separate entity - there being no constant relationship between the two either in symptoms or in response to treatment. Besides the difficulty in initiating movements and the lack of facial expression described above, the effects of akinesia may also be seen in the loss of automatic movements, such as spontaneous gestures, arm-swing when walking, or adaptations of other body-segments to movements of the limbs or head - although the patient may be able to perform these movements to command. Here we have a paradox - whilst automatic movements (which are presumably on a "less conscious" level) are lost - the patient who is unable to walk may be able to dance, and the patient who has difficulty with speech may be able to sing clearly. Obviously further explanation is needed on this point. Akinesia may also suddenly diminish in moments of stress.

The akinesia and rigidity as outlined above further affect the patient's balance and protective mechanisms allowing him to compensate only inadequately for changes in posture, limiting the weight-transference necessary for normal walking and preventing normal balance and equilibrium reactions of the trunk and limbs. The typical gait of the Parkinson's patient is so well known as to need no further description.

\section{Tremor}

In contrast to the akinesia, the tremor does appear to be related to the hypertonus ${ }^{8},{ }^{\theta}$ although not completely dependent upon it. It cannot be thought of as a true "resting", tremor, since it is superimposed upon sustained muscular hypertonus (i.e. sustained postural activity). Furthermore, when hypertonus is sufficiently decreased to allow movement, the tremor frequently disappears. Whilst tremor remains the symptom least affected by both L-dopa and physiotherapy (and stereotaxic surgery shows varying results) this tendency of the tremor to disappear once purposeful activity has been facilitated generally makes it less of a functional problem than might be supposed.

\section{THE APPROACH TO TREATMENT}

Our approach to therapy has been to attempt to diminish rigidity by decreasing $\gamma$ activity and by encouraging coordination of antagonists and also to overcome the akinesia by initiating and facilitating movement. In this way we hope

(i) to assist the vital functions of respiration, feeding and speech

(ii) to re-establish rhythmical co-ordinated movement of trunk and limbs

(iii) to facilitate effective postural and protective responses

(iv) to establish an effective and more normal pattern of locomotion as applied to movement at all stages of the developmental sequence as well as to the walking pattern as such.

(v) to facilitate the re-acceptance of the patient into normal society.

Throughout treatment we have placed much emphasis on rhythm not only in basic limb and trunk movements, but also in changes of position and functional activities. In some cases we have been limited by the general condition of our patients or by the presence of joint contractures. In these cases the necessary precautions were taken and/or additional procedures (such as passive stretching of joints) were undertaken. The majority of patients were, however, able to carry out the type of programme outlined below. 
Patients were treated both individually and in a class. Individual treatment was given with the above aims in view and was directed at intensive rehabilitation of the patient whilst he was hospitalized for stabilization of his L-dopa treatment. Class treatment was instituted at the same stage, both for social and psychological encouragement of the patients, but also, more specifically, to teach the patients exercises which they could perform routinely at home. It is envisaged that some patients may require further periods of intensive individual treatment at some time in the future, perhaps when adjustments of their dosage of L-dopa are necessary, but that the majority will carry out a simple maintenance exercise régime themselves. These patients return every fortnight for a check-up at the clinic, and it was also envisaged that they should take part in a class at each visit, which would afford the physiotherapist the opportunity of not only checking the performance of the exercises, but also of picking out any patient in need of further individual attention. Unfortunately it has not been possible to institute this yet.

\section{INDIVIDUAL TREATMENT}

Very few of our patients have been given any form of cold or heat in an attempt to reduce rigidity prior to exercise sessions, perhaps because we have become aware of the possibility of increased $\gamma$ activity after a latent period, but also because it was felt that the use of ice-packs or towels over an extensive enough area to have much effect was neither justified nor expedient, especially in patients in the older age groups. We do not have a hydrotherapy bath.

\section{Matwork}

Treatment generally began on the mat - the plinth-work later giving the patient a chance to rest before the more strenuous walking exercises. All matwork was done first passively until the rigidity was diminished and rhythmical movement was established. The patient was then encouraged to participate actively, with maximum facilitation and minimum effort so that rhythm and speed of movement were maintained and tone was not increased. In contrast to other authors, who have advocated movement in one direction only initially, we have used reversal patterns from the beginning. Resistance was only introduced when the movement pattern was felt to be completely "free", and was never progressed to maximal resistance. It was felt that this "sub-maximal" resistance was less likely to increase activity.

Head and neck rotation often preceded rolling, in accordance with the normal developmental sequence where head control precedes trunk and limb movements and also to utilize the righting reactions. Thereafter, however, rolling was initiated from the shoulder girdle (and the arm) or the pelvis (and the leg) in deference to the patient's age and possible arthritic changes in the neck. Quick stretch was used at shoulder-girdle and pelvis to initiate the movement, and emphasis was placed upon rotation between shoulder-girdle and pelvis. Movement of the upper arm and leg during rolling was encouraged.

In a similar manner the patient was taken through the stages of rolling to side-lying (elbow-support) or to prone on elbows, of rolling to side-sitting, and of side-sitting to kneeling. Again rhythmical reversals of all movements were done from the beginning, and initial facilitation was followed by only sub-maximal resistance. Neck patterns were often done in the prone-on-elbows position, in order to increase stretch and resistance to the neck extensors. The patterns used were, however, the phasic patterns of Knott and Voss. $^{10}$ It was felt that the neck co-contraction pattern ${ }^{11}$ might further increase the hypertonus. In kneeling, slow rocking (weight transference forwards and backwards) is carried out rhythmically for a multiple purpose. Firstly, by acceleration and deceleration of the head it is hoped to increase mobility via stimulation of the semi-circular canals. ${ }^{11,12}$ In this case proximal mobility will be affected, although as the movement is not in the horizontal plane (in relation to the horizontal semi-circular canal) rotation will be little affected. Secondly, in the kneeling position, stimulation of the carotid sinus may result in reduction of rigidity. Thirdly the joint-compression also tends to reduce hypertonus and lastly, and more obviously, this type of proximal mobility (distal segment fixed) is a prerequisite for the weight transference required for the more skilled action of crawling. Weight transference laterally is also used both as preparation for crawling and in order to facilitate sideways balance reactions. It will have been noted that throughout the matwork the emphasis has been on movement and that static "holding" positions have been avoided. We have, in fact, avoided stabilization techniques almost entirely in view of their tendency not only to further increase hypertonus but also to causé co-contraction of antagonists. It has been felt by several workers that such an isometric contraction of antagonistic muscle groups may be behind the akinesia of Parkinson's disease, causing the patient to "freeze" in one position despite all attempts to move Reciprocal crawling patterns and further progression in mat activities are continued according to the patient's ability. Almost all our patients, even the most severely involved, have been able to follow this type of programme. A balance plank has sometimes been found useful for "rocking" techniques in severely handicapped patients.

\section{Limb movements}

To allow the patient to rest, any passive stretching necessary was then done with the patient comfortably supported on a plinth, prior to using the "rhythm" technique to facilitate limb movements in functional patterns. Scapular patterns were done first, followed by arm and leg patterns The proprioceptive neuromuscular facilitation patterns were used, at first passively and again using reversal patterns from the beginning. As rigidity was reduced, active participation was encouraged by the use of quick stretch and progressed until the pattern could be performed easily against sub-maximal resistance. Bilateral reciprocal (symmetrical) patterns were then used. The exact variation of pattern used was governed both by the patients ability and the functional significance of the pattern, for instance in the legs flexion-adduction-external rotation with knee flexion was reversed to extension-abduction-internal rotation with knee extension. In this way the walking pattern was facilitated without overtaxing the patient's abdominal muscles. In the arms, flexion-adduction-lateral rotation was combined with elbow flexion (for feeding), and extension-abductioninternal rotation with elbow extension (for support). The bilateral asymmetrical "lifting" pattern was also used to encourage trunk rotation with extension.

Functional hand movements, where again the use of rhythm was accentuated, were practised in the class and were not, as a general rule, included in the individual treatment.

\section{Standing and walking}

Of all the areas of treatment in Parkinson's disease, the overlapping influence of various workers can best be seen in the re-education of standing balance and walking. It is even difficult to sort out where one idea or technique ends and another begins - and this is how it should be. Ideas from Rood, the Bobaths, Knott and Voss, Temple Fay and "traditional" régimes can all be combined to the patient's advantage.

Rotation in a swivel chair for stimulation of the horizontal semi-circular canals has been found of great help in facilitating rotational trunk mobility prior to walking. We have used forwards and backwards rocking, of increasing amplitude, in order to facilitate standing up - as advocated by so many other workers - but have avoided pressure on the back of the head as the patient stands up, finding that this tends to cause them to throw their weight backwards and lose their balance. Instead we have tried downwards pressure on the head once the upright position is obtained. Besides being an excellent postural stimulus, the joint compression does appear to release hypertonus, especially in the neck 
flexors where it is often increased by the patients effort to get his body weight forwards and up. Rhythmical lateral weight transference is followed by facilitation of lateral balance reactions. This swinging from foot to foot may also facilitate the tonic lumbar reflex and so aid in the preparation for a reciprocal walking pattern.

Again, no stabilization techniques are used. Anteroposterior weight transference in standing and then in walkstanding is thought to stimulate the Paccinian receptors and so facilitate pick-up, and is therefore progressed immediately to walking facilitated from the hips. In this way it is found that the therapist can control the length of the stride as well as the rhythm and speed. Later, facilitation via the shouldergirdle starts to bring in reciprocal arm movements. Later still these arm movements are facilitated by the use of canes - the patient holding one end of each and the therapist (behind him) the other, initiating the arm movements reciprocally with the leg movements. Chanting or singing often helps the patient to maintain the rhythm. The use of blocks or other obstacles to regulate the length of the step and to teach the patient to lift his feet up, as well as the practising of stopping, starting, changes of direction, steps, etc. are well known to all therapists and need no description; we have certainly not found any reason to change these time-proven techniques.

\section{Vital functions}

Although this aspect of the individual treatment is being described last, it has in practice been incorporated into the treatment at various stages - particularly when the patient needed rest from more strenuous activities. We do, however, feel that we could have done more in this field than we have done. We have used breathing exercises, utilizing quick stretch at the end of expiration, to increase vital capacity and thoracic mobility, but have not tried Rood's method, which might well better encourage reciprocal action between the diaphragm and the abdominal muscles. We have also used vinegar (or where necessary ammonia) to activate a protective facial response in cases of severely mask-like face. In milder cases we have used brisk rubbing with ice and quick stretch to activate the facial muscles. One of the privileges of treating patients on L-dopa has been to see those facial movements obtained during treatment carried over to automatic facial expressions during daily activities; previously one of the most difficult things to achieve.

Looking back, we feel that we should have done more for sucking, swallowing, tongue movements and speech - our only excuse being that we were overwhelmed by the sudden invasion of almost 20 Parkinson's patients at the beginning of the year! Another problem which affects some patients due to the use of drugs which are parasympatholytic agents - is difficulty in voiding, both in initiating voiding and in a high residual. Although our patients on L-dopa have ceased their previous medications, one or two still experienced this problem and it is possible that we could have helped here also.

\section{CLASS TREATMENT}

As mentioned before, the class treatment was aimed (on the physical side) at teaching patients exercises for prolonged home use.

These had, therefore, to be simple, but included easy mat (or floor) activities such as rolling, bridging and back extension as well as other exercises. The back extension exercise (in supine) was one of the few occasions on which an isometric contraction was called for - in an attempt to increase $\gamma$ in back extensor muscles stretched and weakened by the habitually flexed posture. Exercises for shoulder girdle and thoracic mobility, pendular arm exercises (sometimes holding a moderately light object to increase momentum) and trunk rotation (sometimes combined with flexion and extension) were taught in sitting, as well as rhythmical, functional hand movements such as tearing or folding paper, dealing cards, "counting" money and rolling squeezing silicone putty. Exercises in standing were included as the patients progressed, accentuating, large trunk movements (especially rotatory stretching movements), "marking time" and marching. General agility exercises such as bicycling or jogging became possible for most patients.

Since the scheme of home exercises was, of necessity, rather long, the patients were instructed and corrected intensively whilst in hospital. We found that many patients would, of their own accord, practice their class exercises alone in the ward and it was these patients who made the best progress.

In addition to a scheme of exercises, patients were given general advice such as rocking to stand up; doing exercises after a warm or cool bath, practising facial movements in front of a mirror and; above all, remaining active at all costs. Several returned to their former occupations.

\section{SUMMARY}

An approach to physiotherapy as used for patients with Parkinson's disease being treated with L-dopa at the Karl Bremer Hospital, Cape Province, has been outlined. The aim was to show how neurophysiologically based techniques could be combined with more traditional methods in the overall treatment programme. It is felt that the programme as outlined could be further improved upon and that the advent of L-dopa has, far from limiting the role of physiotherapy in the treatment of Parkinson's disease, greatly increased its possibilities.

\section{ACKNOWLEDGEMENTS}

Thanks are due to Dr. A. van Wieringen, for his referral of the patients, to the staff of the Physiotherapy Department at Karl Bremer Hospital for allowing the students to treat these patients, and to the students for their enthusiasm in carrying out the treatment programmes.

\section{REFERENCES}

1. Poskanzer and Schwab, in: Gillingham, F. J., and Donaldson, I. M. L. (Eds.), Proc. Third Symposium on Parkinson's Disease, Edinburgh, Livingstone and Co., 1969.

2. Knott, M. Report of a Case of Parkonsonism treated with Proprioceptive Facilitation Technics, Phys. Ther. Rev. 37, 4, April, 1957.

3. Alexander, B. Parkinson's Disease: Recent Trends in Physiotherapy. Aust. J. Physiother., 16, 2, June, 1970.

4. Voss, D. E. Proprioceptive Neuromuscular Facilitation, in: An Exploratory and Analytical Survey of Therapeutic Exercise, Am. J. Phys. Med., 46, 1, Feb., 1967.

5. Stevens, I. H. K., and Roche, G. D. Management of Parkinson's Disease, Ann. Phys. Med. 1, 8, Oct., 1953.

6. Murray, W. Parkinson's Disease, Aspects of Functional Training, Phys. Ther. Rev., 36, 9, Sept., 1956.

7. Bilowit, D. S. Establishing Physical Objectives in the Rehabilitation of Patients with Parkinson's Disease, Phys. Ther. Rev., 36, 3, March, 1956.

8. Spiegel, E. A. Problems in Parkinson Research regarding the Mechanisms of Rigor, Akinesia and Tremor, Chap. 19 in: Symposium on Parkinson's Disease, ed. Barbeau et $a l$, New York and London, Grune and Stratton, 1965.

9. Cooper, I. S. Relationship of Cerebellar Intention Tremor to Resting Tremor of Parkinson's J. Am. Ger. Soc., 14, 3, March, 1966.

10. Knott, M., and Voss, D. Proprioceptive Neuromuscular Facilitation, New York, Harper-Row, Publ. 1966.

11. Stockmeyer, S. A. An Interpretation of the Approach of Rood to the Treatment of Neuromuscular Dysfunction, in: An Exploratory and Analytical Survey of Therapeutic Exercise, Am. J. Phys. Med., 46, 1, Feb., 1967.

12. Rood, M. S. Unpublished notes, S.A.S.P. Post-graduate course, Johannesburg, July-August, 1969.

13. Stern, P. H., McDowell, F., Miller, J. M., and Robinson, M. Levadopa and Physical Therapy in Treatment of Patients with Parkinson's Disease. Arch. Phys. Med. and Rehab., 51, 5, May, 1970. 